\title{
EVALUACIÓN DE LAS INCIDENCIAS DE SALINIDAD Y pH SOBRE LA BIOMASA, PRODUCTIVIDAD Y ACUMULACIÓN DE LÍPIDOS EN CULTIVOS DE Chlorella vulgaris EN UN FOTOBIORREACTOR DE PLACA PLANA
}

\author{
EVALUATION OF THE INCIDENCES OF SALINITY AND PH ON \\ THE BIOMASS, PRODUCTIVITY AND LIPIDS ACCUMULATION \\ IN CULTURES OF Chlorella vulgaris IN A FLAT PLATE \\ PHOTOBIOREACTOR
}

\author{
Diego Rubio-Fernández \\ M. Sc. Biología \\ Fundación Universidad América \\ Bogotá. Colombia \\ diego.rubio@profesor.uamerica.edu.co
}

\author{
Gregorio Alejandro Hernández \\ Ingeniero Químico \\ Fundación Universidad América \\ Bogotá. Colombia \\ alejo0390@gmail.com
}

\begin{abstract}
Resumen- La presente investigación evaluó la incidencia de la salinidad, pH y productividad en un cultivo de Chlorella vulgaris en un fotobiorreactor de placa plana, la evaluación de las incidencias se hizo teniendo en cuenta tres criterios de selección, pero antes de llegar a los tres criterios se hizo una fase de selección de condiciones teniendo en cuenta cuatro concentraciones de salinidad y cuatro rangos $\mathrm{pH}$ obtenidos en estudios anteriores, al tener en cuenta las curvas de crecimiento, la productividad volumétrica y el análisis estadístico se seleccionan las dos mejores condiciones de salinidad y nivel de $\mathrm{pH}$ y son llevadas al fotobiorreactor en donde se selecciona la mejor condición para determinar el perfil de ácidos grasos y encontrar la debida aplicación.
\end{abstract}

Palabras clave- Chlorella vulgaris, fotobiorreactor, lípidos, pH, productividad, salinidad.

Abstract- The present research evaluated the incidence of Salinity, pH and productivity in a culture of ChloreIla vulgaris in a flat plate photobioreactor, the evaluation of the incidences was made based on three aspects, but, before this a previous selection of the culture conditions was made from the test of four salinity concentrations and four $\mathrm{pH}$ ranks obtained from previous studies and based on the growth curves, the volumetric productivity and the statistical analysis we selected the two better conditions of salinity and $\mathrm{pH}$ in order to develop cultures on a photobioreactor level where the best growth curve was selected and analyzed looking for a fatty acids profile and define the proper application.

Keywords- Chlorella vulgaris, photobioreactor, lipids, ph, productivity, salinity.

\section{INTRODUCCIÓN}

La producción de lípidos ha despertado el interés para la investigación debido a sus propiedades, y las diversas formas de obtenerlos han tomado un rol importante en la industria química. Las diferentes aplicaciones de estas biomoléculas como alimentos, fármacos y especialmente biocombustibles han generado la búsqueda por nuevas fuentes entre las que se encuentran las microalgas.

Adicionalmente, se está desarrollando un enfoque interesante para suplir la inmensa demanda de las nuevas formas de energías más limpias y renovables, cuya generación de impacto ambiental sea reducida. La búsqueda de nuevas tecnologías para la producción de biocombustibles es un nuevo punto de partida para la biotecnología 
y el desarrollo de nuevas condiciones iniciales de producción, la búsqueda y el uso de nuevas cepas de microorganismos, en este caso microalgas, cuyas ventajas con respecto a los demás microorganismos son notorias; entre estas se encuentran el tiempo de crecimiento, adaptación a cambios en el medio de cultivo, absorción de $\mathrm{CO}_{2}$ y, en especial, su capacidad de asimilar energía solar para convertirla en biomasa. Adicionalmente, no son microorganismos selectivos por lo cual el medio de cultivo puede ser desde aguas residuales, hasta fertilizantes para producción de lípidos y otras sustancias de alto valor agregado.

En las últimas décadas se han desarrollado sistemas en los que se evalúa el crecimiento microalgal, que son los fotobiorreactores, los cuales, según su diseño, permiten la optimización de diferentes condiciones como la transfencia de luz y la transferencia de masa buscando la posibilidad de escalamiento[1].

Esta investigación se enfocó en el diseño de un tipo de fotobiorreactor de placa plana debido a las ventajas que presenta con respecto a los demás, una de ellas es la recepción de luz gracias al área de sus placas laterales y su relación superficievolumen [2].

Siendo los fotobiorreactores sistemas en donde se pueden manipular condiciones de cultivo, deben seguir aspectos esenciales para su buen funcionamiento como la trayectoria de luz, aireación, el pH y longitud de la tubería de aireación. Adicionalmente los fotobiorreactores de panel plano tienen una relación superficie-volumen que permite una eficiente transmisión de la luz al compararse con los fotobiorreactores cilíndricos.

Anteriormente se han desarrollado investigaciones en las que se evaluaron condiciones específicas; una de ellas es la variación de las concentraciones de un agente salino en un cultivo, con el fin de comparar la biomasa obtenida con otros cultivos [3]with $420 \mathrm{mM}$ of total salts. Specific growth rates of $0.08 \mathrm{~h}(-1$. Otra condición esencial es el nivel de $\mathrm{pH}$ ya que al variar estos niveles en un cultivo favorecen en la productividad volumétrica y en la obtención de ácidos grasos específicos [4]. Teniendo en cuenta la importancia de los factores $\mathrm{pH}$ y salinidad para los cultivos de microal- gas a escala de laboratorio, el presente estudio se enfocó en buscar los rangos adecuados para implementar cultivos a nivel de fotobiorreactor.

\section{MATERIALES Y MÉTODOS}

\section{Especie de microalga cultivada}

Para el desarrollo experimental se utilizó la microalga Chlorella vulgaris suministrada por el Laboratorio de Algas de la Universidad Nacional de Colombia. Esta microalga hace parte de la familia de las Cloroficeas o algas verdes.

El desarrollo de este artículo se enfocó en 2 fases, en las que se determinaron las condiciones adecuadas de cada variable (salinidad y $\mathrm{pH}$ ) y que se distribuyeron de la siguiente manera:

- Fase de selección de condiciones.

- Desarrollo experimental.

\subsection{Fase de selección de condiciones}

Para cumplir con este ítem se desarrollaron cuatro cultivos por cada variable por estudiar, en donde se seleccionaron las concentraciones salinas y los niveles de $\mathrm{pH}$ mediante investigaciones realizadas anteriormente. Las cuatro concentraciones salinas fueron $0.1,0.2,0.3$ y $0.4 \mathrm{M}$ [5]. El montaje para este cultivo se hizo mediante cuatro erlenmeyers cada uno de $250 \mathrm{~mL}$, donde se distribuyó la microalga (Chlorella vulgaris) con respectiva concentración salina, se almacenó en una incubadora previamente limpia para evitar que agentes ajenos al cultivo afectaran su crecimiento [6]. El sistema de aireación fue proporcionado por una bomba de cuatro salidas cuyo caudal es de $3.4 \mathrm{~L} /$ min y una potencia de $8 \mathrm{~W}$ con el fin de evitar una sedimentación del cultivo [7], la iluminación del cultivo fue abastecida por una lámpara de halógeno de color rojo de una potencia de $8 \mathrm{~W}$ con el fin de que los cultivos realicen el proceso de la fotosíntesis, El fotoperiodo consistió en 12 horas de luz y 12 horas de oscuridad [8], para el control de las horas de luz se instaló un temporizador que impide que el cultivo reciba más de las 12 horas de luz establecidas, ya que al no tener controlado las horas de intensidad lumínica el cultivo se ve afectado por fotoinhibición. Dicho montaje se puede apreciar en la Fig. 1. 
Fig. 1. MONTAJE PRIMERA FASE

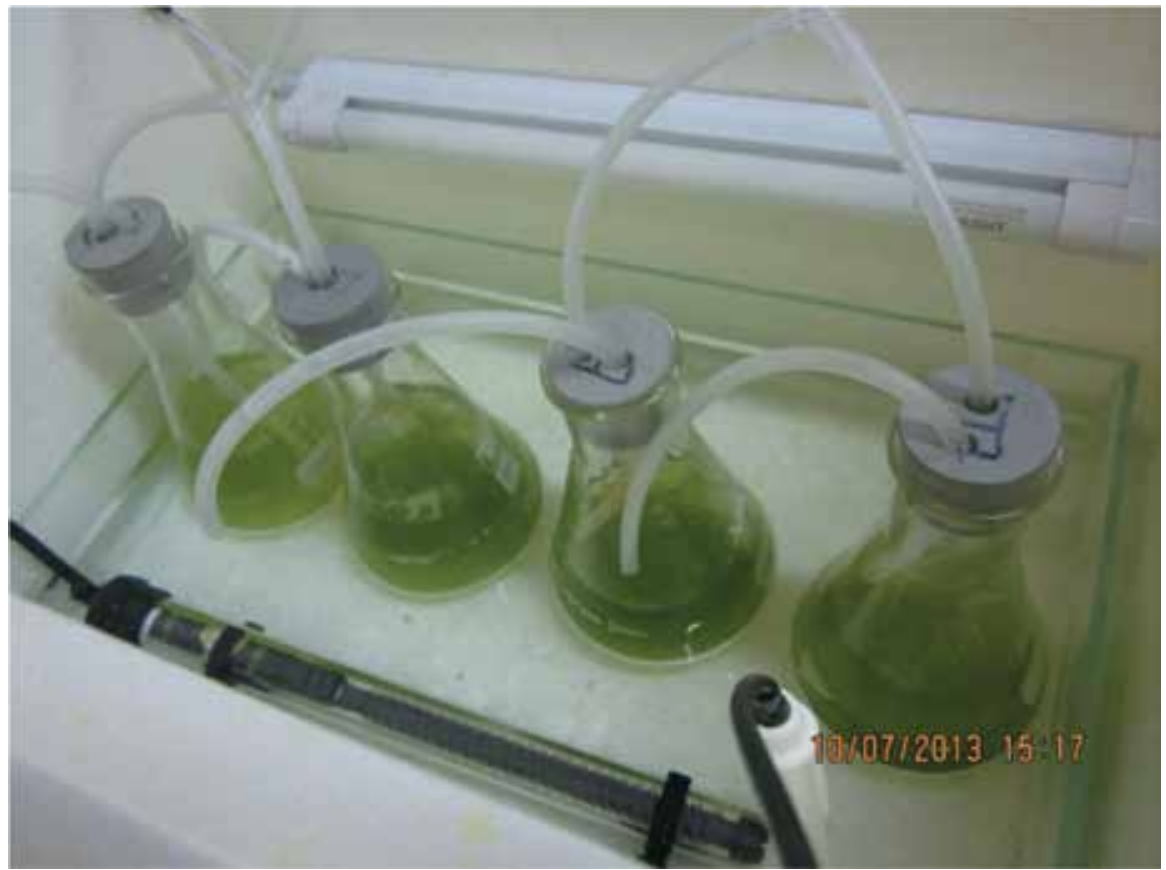

Fuente: autores

\subsubsection{Sistema de control de temperatura}

El sistema de control de la temperatura consistió en un termostato acoplado a una resistencia graduable; este sistema permitió definir rangos de temperatura necesarios para el cultivo, en este caso entre $22^{\circ} \mathrm{C}$ y $24^{\circ} \mathrm{C} \pm 1^{\circ} \mathrm{C}$. Para su instalación se diseñó un recipiente de vidrio en el que se vertió agua. Con el termostato sumergido este calienta el agua a una temperatura de $25^{\circ} \mathrm{C}$ [9] que es la adecuada para el crecimiento celular del cultivo.

En la primera fase se seleccionaron dos condiciones que presentaron cambios significativos para cada condición estudiada en esta investigación, es decir dos concentraciones de salinidad y dos niveles de pH. Para llegar a ello se compararon las curvas de crecimiento y la productividad por análisis estadístico ANOVA.

Para calcular la concentración celular de la cámara de Neubauer se empleó la ecuación 1. [10]
Donde el volumen de la cámara es de $0.1 \mathrm{~mm}^{3}$, los cuadrantes totales son 25 y la concentración inicial de cultivo para cada ensayo es de $2.0 \times 106$ células por mililitro.

Para determinar la curva de crecimiento por el método espectrofotométrico se determinó la curva espectral que definió el valor de $750 \mathrm{~nm}$ como valor de lectura para el seguimiento del cultivo. Adicionalmente, se verifico la capacidad del espectofotómetro para medir la concentración de microalgas por la curva de calibración, y por último, la curva de crecimiento se obtuvo para determinar las respectivas curvas (ecuación 4).

Las ecuaciones matemáticas utilizadas fueron:

$$
C 1=\frac{C p}{A p} A m
$$

Donde $\mathrm{C}_{1}$ es la concentración corregida, $\mathrm{C}_{\mathrm{p}}$ es la concentración patrón, $A_{p}$ es la absorbancia patrón y $A_{m}$ es la absorbancia de la muestra.

$$
W * C 1=A^{*}
$$

$$
\frac{\text { Células }}{m L}=\frac{n \text { úmero de células } * \text { factor de dilució } n}{\text { volúmen de la cámara } *(\text { cuadrantes contados } / \text { cuadrantes totales })} * \frac{1000 \mathrm{~mm}^{3}}{1 \mathrm{~mL}}
$$


Donde w es la fracción de ajuste y $A^{*}$ es la absorbancia corregida

$$
A=\in * b * c m
$$

Donde $\varepsilon$ es el coeficiente de absorción, b es el ancho de la celda y $\mathrm{cm}$ es la concentración de la muestra.

La productividad volumétrica que es el segundo criterio de selección se determinó por medio de la siguiente ecuación:

$$
\text { Productividad }=\frac{\text { Biomasa Seca }}{\text { Volumen de cultivo } * \text { Días de cultivo }}(5)
$$

Donde la biomasa seca es la cantidad en gramos obtenida del cultivo y las unidades del volumen de cultivo es en litros.

Con el objeto de definir si se presentaron diferencias significativas a nivel de las concentraciones celulares finales obtenidas en los diferentes tratamientos, se empleó un Análisis de Varianza ANOVA [11] de un solo factor.

Los niveles de $\mathrm{pH}$ que se utilizaron en el cultivo fueron $6.5,7,7.5$ y 8.3 [12], que también fueron cultivados cada uno en un Erlenmeyer de $250 \mathrm{~mL}$. Las soluciones de $\mathrm{pH}$ fueron preparadas mediante la graduación de $\mathrm{HCl}$ y $\mathrm{NaOH}$. Ahora bien, se utilizaron los tres criterios para determinar los niveles de $\mathrm{pH}$ adecuados, con la diferencia de que con el análisis estadístico se realizó la prueba Tuckey. Los valores de $\mathrm{pH}$ fueron tomados diariamente para verificar que se encontrara dentro de los rangos requeridos para el respectivo tratamiento experimental. Estos valores de $\mathrm{pH}$ se mantuvieron estables durante las fases de crecimiento de los cultivos microalgales según el tratamiento experimental.

\subsection{Desarrollo experimental}

Con las condiciones previamente seleccionadas, se cultivan en un sistema de producción de biomasa o fotobiorreactor cuyo material es de PMMA (polimetil-metacrilato) [13] el cual presenta propiedades que son adecuadas para el cultivo, tales como la transparencia, la resistencia química y la resistencia a la presión de fluidos. El tipo de fotobiorreactor en el que se trabajó fue el de placa plana debido a que tiene un parámetro que es primordial para el ensamblaje del equipo y es la relación superficie volumen, el cual permite determinar si el equipo es adecuado para el proceso fotosintético [14] dicho parámetro fue estimado mediante la ecuación:

$$
\text { Relación Sup }- \text { Vol }=\frac{\text { Área de la placa lateral }}{\text { Volumen de equipo }}
$$

Inicialmente se adquieren dos láminas de PMMA de dimensiones de $40 \times 20 \mathrm{~cm}$ y de $8 \mathrm{~mm}$ de espesor, estas vienen siendo las caras laterales del equipo, las caras frontales son láminas de dimensiones de $2 \times 20 \mathrm{~cm}$ y $8 \mathrm{~mm}$ de espesor, estas piezas son ensambladas mediante cloruro de metilo que permite que las piezas se mantengan rígidas durante el pegado. Las piezas superiores $\mathrm{e}$ inferiores constan de dimensiones de $2 \times 40 \mathrm{~cm}$ con la diferencia de que la placa superior tiene un orificio de $5 \mathrm{~mm}$ el cual permite la desgasificación del cultivo, ya que de no ser así el cultivo se satura de gas, provocando cambios en el cultivo. Para el cierre del fotobiorreactor se hicieron 6 orificios a la placa superior y se insertaron 6 tornillos. Se le adiciona caucho en la parte superior del fotobiorreactor para facilitar su cierre, logrando así que no se afecten las paredes laterales, ya que el exceso de fuerza al cerrar el equipo puede provocar que el material se quiebre.

El sistema de control de temperatura fue proporcionado por un termostato para mantener el cultivo a una temperatura de $250 \mathrm{C}$, pero con la diferencia de que fue instalado en una de las paredes del equipo. El sistema de agitación fue proporcionado por una bomba centrifuga $\mathrm{RICOH}$ de $1 / 2$ HP de potencia, el aire es transportado a unas mangueras de $1 / 4$ de pulgada para luego terminar en el tubo de aireación del equipo que fue ubicado en la parte inferior de este para generar el burbujeo manteniendo el equipo en constante agitación.

Se pone en marcha el fotobiorreactor, sembrando la microalga en el medio de cultivo dentro del fotobiorreactor, la siembra se realiza para cada una de las concentraciones, donde la concentración inicial de cultivo es de $2.0 \times 10^{6}$ células por mililitro, haciendo 2 réplicas por muestra que se dejan en el equipo por 19 días. Cada uno de los procedimientos mencionados a continuación se hace para todos los ensayos y sus respectivas réplicas, dicho montaje se muestra en la Fig. 2. 
Fig. 2. FOTOBIORREACTOR DE PANEL PLANO USADO EN LA EXPERIMENTACIÓN

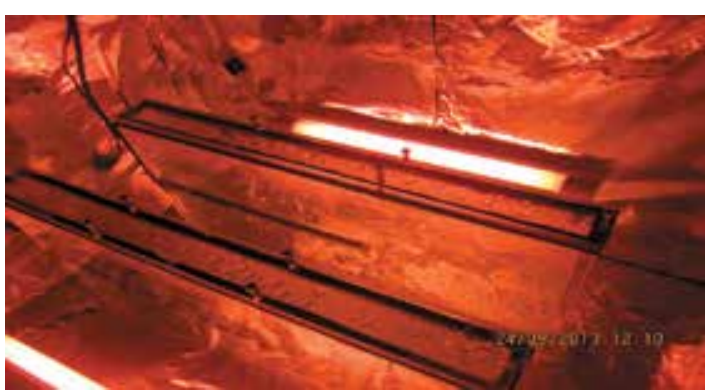

Fuente: autores.

Las réplicas de cada ensayo permanecen en el fotobiorreactor por 19 días, en los cuales se hacen conteos celulares directos cada 24 horas, similares a los realizados anteriormente en los ensayos preliminares, lo que permite evaluar las curvas de crecimiento de cada concentración.

Luego de culminar el cultivo se seleccionaron las dos condiciones que cumplieron con los tres criterios, y se liofilizaron para mantener la cantidad lipídica de cada muestra, ver Fig. 3.

Fig. 3. BIOMASA CONCENTRADA DESPUÉS DE CENTRIFUGACIÓN
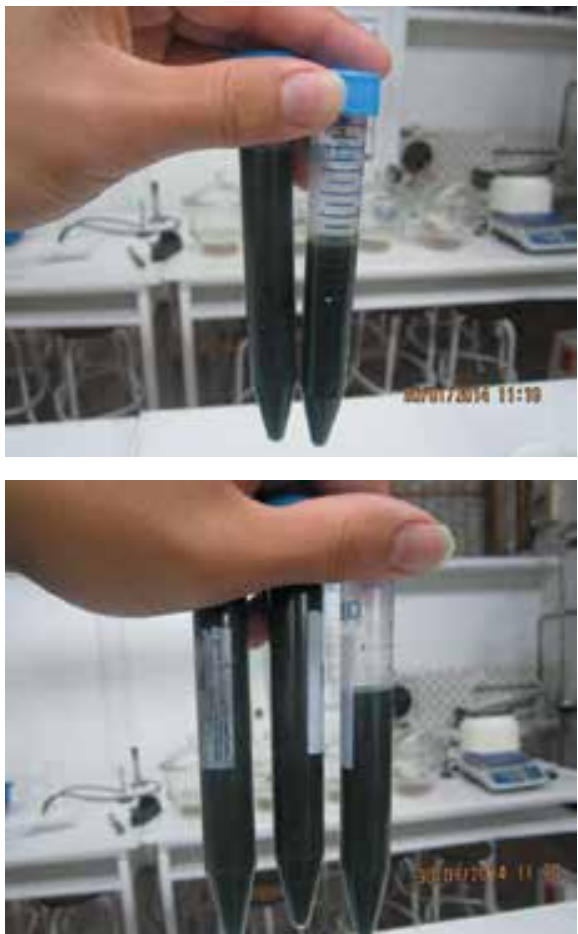

Fuente: autores.

Las muestras descartadas fueron centrifugadas a una velocidad de 4000 rpm durante $10 \mathrm{~min}$ [15], permitiendo que se obtenga una biomasa concentrada como lo muestra la Fig. 4.
Fig. 4. BIOMASA LIOFILIZADA
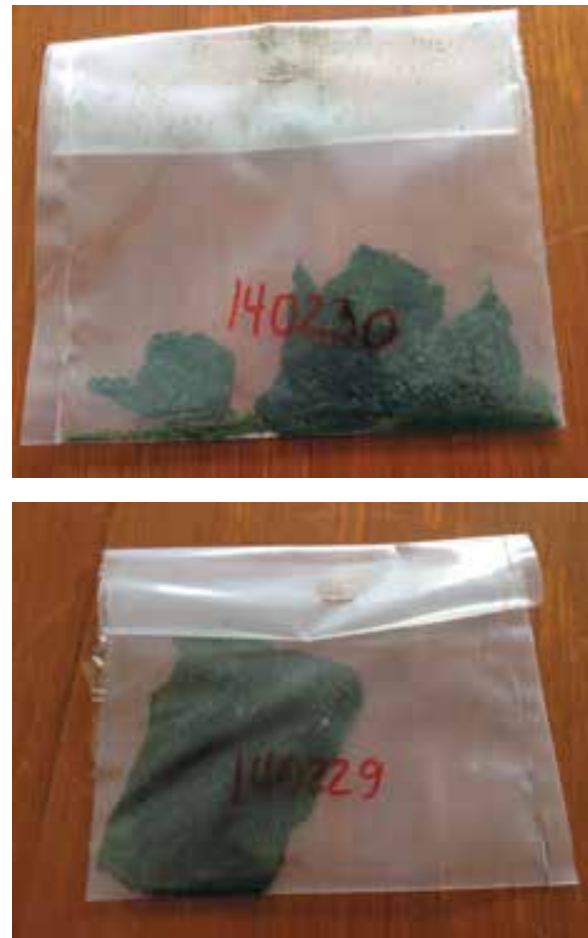

Fuente: autores.

Luego las muestras concentradas son llevadas a un crisol. El crisol que contiene la biomasa húmeda se lleva a un horno durante 3 horas y media a una temperatura de $60^{\circ} \mathrm{C}$. Con ese tiempo se obtuvo la biomasa seca y así se pudo determinar la productividad de las muestras descartadas.

\section{RESULTADOS}

Utilizando la ecuación (1) se obtuvieron las curvas de crecimiento para las cuatro concentraciones salinas que se reportaron en la Fig. 5.

Fig. 5. CURVAS DE CRECIMIENTO A DIFERENTES CONCENTRACIONES SALINAS

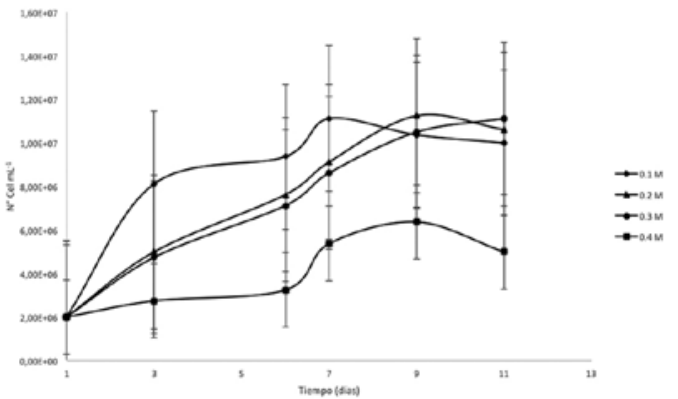

Fuente: autores. 
Como se muestra en la Fig. 5 las condiciones que no presentaron problemas en su adaptación son los cultivos de las concentraciones de 0.2 y $0.3 \mathrm{M}$, alcanzando un máximo de concentración celular de 1.12 x $10^{7}$ y 1.11 x $10^{7}$, respectivamente, cumpliendo así con el primer criterio.

Para determinar la curva por espectrofotometría se utilizaron las ecuaciones (2, 3 y 4 ) en cada uno de las diferentes concentraciones salinas. Los resultados fueron reportados en la Fig. 6.

Fig. 6. CURVA DE CRECIMIENTO CONCENTRACIONES SALINAS

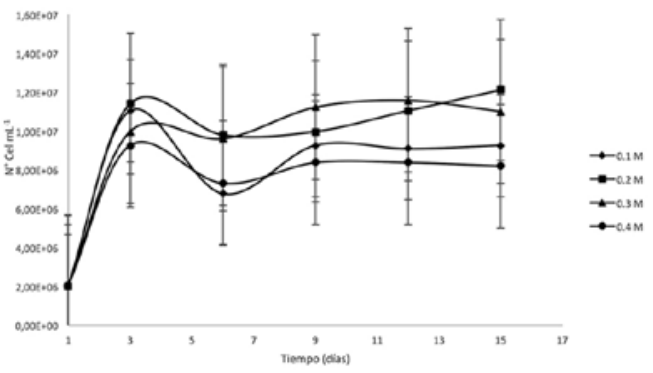

Fuente: autores.
Al comparar las gráficas realizadas por estos dos métodos de conteo, su forma y valores difieren, pero aun así coinciden con la selección de las condiciones adecuadas, siendo las concentraciones de 0.2 y $0.3 \mathrm{M}$ las que mayor concentración celular presentaron.

La productividad fue determinada por la ecuación (5) los resultados se muestran en la Tabla I

Una vez calculada la productividad, la Tabla II muestra que las concentraciones salinas de $0.2 \mathrm{y}$ $0.3 \mathrm{M}$ son las adecuadas para el cultivo en el fotobiorreactor mientras que las concentraciones de 0.1 y $0.4 \mathrm{M}$ quedan descartadas, por ahora los dos primeros criterios han coincidido en la selección.

El análisis estadístico fue empleado por una Tabla ANOVA de un factor donde se compara por medio del crecimiento celular, cuál de las concentraciones presentan cambios significativos. Los resultados que se reportaron en el análisis muestran que las concentraciones salinas de 0.2 y 0.3 $\mathrm{M}$ presentan cambios significativos coincidiendo con los demás criterios.

TABLA. I

PRODUCTIVIDADES A DIFERENTES CONCENTRACIONES SALINAS

\begin{tabular}{|c|c|c|c|c|c|}
\hline $\begin{array}{c}\text { Concentración } \\
{[\mathrm{M}]}\end{array}$ & $\begin{array}{c}\text { Peso húmedo } \\
{[\mathrm{g}]}\end{array}$ & $\begin{array}{c}\text { Peso seco } \\
{[\mathrm{g}]}\end{array}$ & $\begin{array}{c}\text { Peso biomasa seco } \\
{[\mathrm{g}]}\end{array}$ & $\mathbf{g} / \mathbf{L}$ & $\begin{array}{c}\text { Productividad } \\
\text { g/Ldía }\end{array}$ \\
\hline 0,1 & 2,625 & 2,122 & 0,045 & 0,225 & 0,019 \\
\hline 0,2 & 2,87 & 1,157 & 0,090 & 0,451 & 0,038 \\
\hline 0,3 & 3,331 & 1,16 & 0,093 & 0,465 & 0,039 \\
\hline 0,4 & 1,813 & 1,139 & 0,026 & 0,128 & 0,011 \\
\hline
\end{tabular}

Fuente: autores.

TABLA II

ANOVA PARA ANÁLISIS DE LOS TRATAMIENTOS DE SALINIDAD

\begin{tabular}{|c|c|c|c|c|c|c|}
\hline $\begin{array}{c}\text { Origen de las } \\
\text { variaciones }\end{array}$ & $\begin{array}{c}\text { Suma de cuadrados } \\
{\left[10^{13}\right]}\end{array}$ & $\begin{array}{l}\text { Grados de } \\
\text { libertad }\end{array}$ & $\begin{array}{c}\text { Promedio de } \\
\text { los cuadrados } \\
{\left[10^{12}\right]}\end{array}$ & $F$ & Probabilidad & $\begin{array}{c}\text { Valor crítico } \\
\text { para } F\end{array}$ \\
\hline Entre grupos & 1,644 & 3 & 5,481 & 1,532 & 0,237 & 3,098 \\
\hline Dentro de los grupos & 7,155 & 20 & 3,577 & & & \\
\hline Total & 8,799 & 23 & & & & \\
\hline
\end{tabular}

Fuente: autores. 
La Tabla Anova para los tratamientos de salinidad no muestra diferencias significativas entre los tratamientos de salinidad, es decir, las diferentes concentraciones en el medio de cultivo. Para los niveles de $\mathrm{pH}$ se utilizaron las mismas ecuaciones para construir las curvas de crecimiento tanto por conteo de células así como por espectrofotometría, dichos resultados se reportaron en las Figs. 7 y 8.

Fig. 7. CURVA DE CRECIMIENTO A DIFERENTES NIVELES DE PH

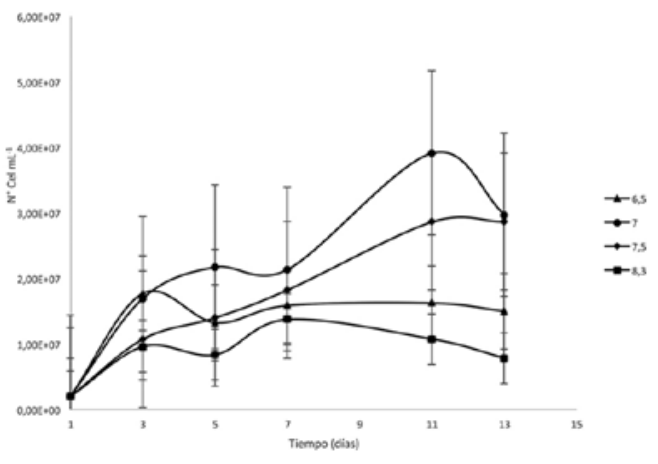

Fuente: autores.

Fig. 8. CURVA DE CRECIMIENTO DE DIFERENTES NIVELES DE PH

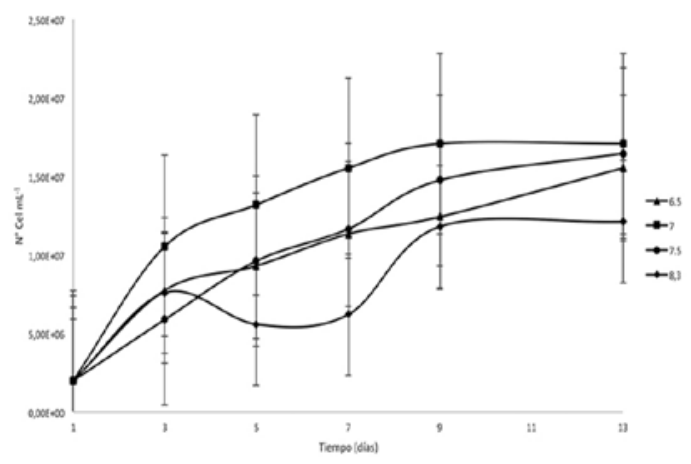

Fuente: autores.

En la gráfica las condiciones adecuadas son los niveles de $\mathrm{pH}$ de 7 y 7.5 con una concentración de 3.91 x $10^{7}$ y 2.86 x $10^{7}$ células por mililitro, mientras que en la gráfica las condiciones adecuadas para el cultivo fueron los niveles de $7 \mathrm{y}$ 7.5 , respectivamente, ya que presentaron una mayor concentración celular en el día 9 de $1.71 \mathrm{x}$ $10^{7}$ células por mililitro para el pH 7 , y en el día 13 de $1.65 \times 10^{7}$ células por mililitro, comparando las gráficas obtenidas los niveles de $\mathrm{pH}$ adecuados tanto para la cámara de neubauer como para espectrofotometría son los niveles de 7 y 7.5 , lo que confirma la similitud de las dos mejores condiciones en estos dos métodos aplicados a la hora de seleccionar las condiciones.
La productividad volumétrica de cada nivel de pH se calculó mediante la ecuación (5), los valores fueron reportados en la Tabla III.

TABLA III

PRODUCTIVIDADES A DIFERENTES NIVELES DE pH

\begin{tabular}{|c|c|c|c|c|c|}
\hline $\begin{array}{c}\text { Rango } \\
\text { de pH }\end{array}$ & $\begin{array}{c}\text { Peso } \\
\text { húmedo } \\
\text { [g] }\end{array}$ & $\begin{array}{c}\text { Peso } \\
\text { seco } \\
\text { [g] }\end{array}$ & $\begin{array}{c}\text { Peso } \\
\text { biomasa } \\
\text { seco [g] }\end{array}$ & g/L & Productividad \\
\hline 6.0 & 2,622 & 1,11 & 0,043 & 0,172 & 0,014 \\
\hline 7.0 & 2,870 & 1,18 & 0,113 & 0,452 & 0,038 \\
\hline 7,5 & 3,331 & 1,03 & 0,046 & 0,184 & 0,015 \\
\hline 8,3 & 1,813 & 1,09 & 0,023 & 0,092 & 0,008 \\
\hline
\end{tabular}

Fuente: autores.

Los resultados obtenidos muestran que los niveles de $\mathrm{pH}$ adecuados fueron 7 y 7.5 coincidiendo con la selección hecha por las curvas de crecimiento. En cambio, los niveles de 6 y 8.3 quedan descartados debido a su baja productividad y concentración celular.

En el análisis estadístico para determinar los cambios significativos de cada uno de los niveles de pH no se utilizó la Tabla ANOVA de un factor, debido a que todos los niveles de pH presentaron cambios significativos y no se llegaría a una selección de condiciones. Para ello se utilizó otro análisis estadístico el método (C. W. Dunnet) en el que se compararon los datos alcanzados en cada uno de los niveles de $\mathrm{pH}$ con un control, obteniendo cambios significativos en los niveles de 7 y 7.5 comprobando una vez más que los tres criterios de selección coinciden, Tabla IV.

La Tabla IV sugiere que no se presentaron diferencias significativas para la productividad entre los diferentes tratamientos pre-experimentales para $\mathrm{pH}$. Con las condiciones seleccionadas (salinidad: 0.2 y $0.3 \mathrm{M}$ y pH: 7 y 7.5 ) se cultivaron en un fotobiorreactor de placa plana, donde se calculó la relación superficie-volumen utilizando la ecuación (6) donde se obtuvo un valor de $50.11 \mathrm{~m}^{-1}$; según una investigación realizada el rango adecuado de la relación superficie-volumen para construir un fotobiorreactor de placa plana está entre los 20 y $200 \mathrm{~m}^{-1}$, lo cual indica que el equipo es el adecuado para el cultivo de microalgas [16], [17]. 
TABLA IV

ANOVA PARA ANÁLISIS DE TRATAMIENTOS DE pH

\begin{tabular}{|c|c|c|c|c|c|c|}
\hline $\begin{array}{c}\text { Origen de las } \\
\text { variaciones }\end{array}$ & $\begin{array}{c}\text { Suma de } \\
\text { cuadrados }\end{array}$ & $\begin{array}{c}\text { Grados de } \\
\text { libertad }\end{array}$ & $\begin{array}{c}\text { Promedio de } \\
\text { los cuadrados }\end{array}$ & $\mathbf{F}$ & Probabilidad & $\begin{array}{c}\text { Valor crítico } \\
\text { para F }\end{array}$ \\
\hline Entre grupos & $5,398 \mathrm{E}+13$ & 3 & $1,800 \mathrm{E}+13$ & 2,017 & 0,138 & 3,009 \\
\hline $\begin{array}{c}\text { Dentro de los } \\
\text { grupos }\end{array}$ & $2,141 \mathrm{E}+14$ & 24 & $8,921 \mathrm{E}+12$ & & & \\
\hline Total & $2,681 \mathrm{E}+14$ & 27 & & & & \\
\hline
\end{tabular}

Fuente: autores.

Ahora bien, el volumen de cultivo fue de $1.4 \mathrm{~L}$ y el método de conteo que se seleccionó fue espectrofotometría las curvas de crecimiento de las concentraciones salinas y los niveles de $\mathrm{pH}$ fueron calculadas mediante las ecuaciones $(2,3, y$ 4), respectivamente y manteniendo un $\mathrm{pH}$ de 7 , obteniendo las Fig. 9 y Fig. 10.

Fig. 9. CURVA DE CRECIMIENTO DE CONCENTRACIÓN SALINA DE $0.2 \mathrm{M}$

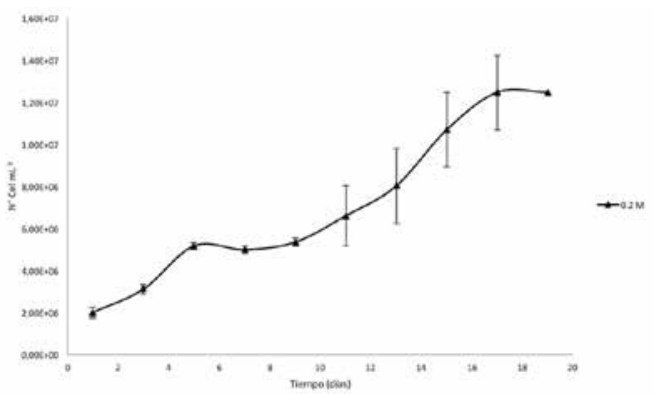

Fuente: autores.

Fig. 10. CURVA DE CRECIMIENTO DE CONCENTRACIÓN SALINA DE 0.3 M

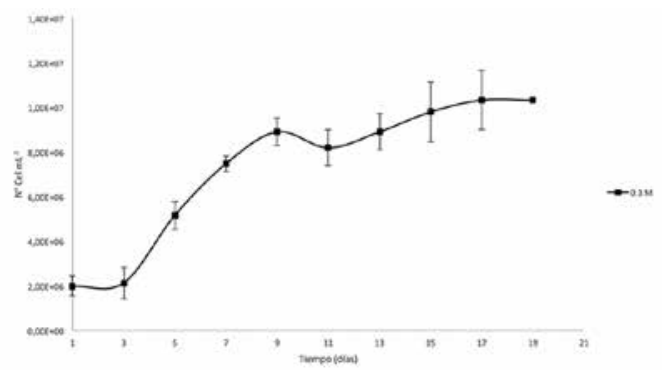

Fuente: autores.

Al comparar las gráficas tenemos que la que mejor se adecua a las condiciones salinas es la del cultivo con la concentración de $0.2 \mathrm{M}$, esto muestra que la microalga es poco halotolerante [18], es decir, que a medida que se incrementa la concentración salina afecta el crecimiento celular disminuyendo su concentración.

Los resultados obtenidos a nivel del crecimiento de los cultivos, para el factor $\mathrm{pH}$, sugieren que la concentración microalgal es más alta a pH neutro, aunque la microalga haya tenido una fase de adaptación larga de nueve días, el día décimo la microalga creció a unas concentraciones considerables, Fig. 11 y Fig. 12.

Fig. 11. CURVA DE CRECIMIENTO DE NIVEL DE PH 7

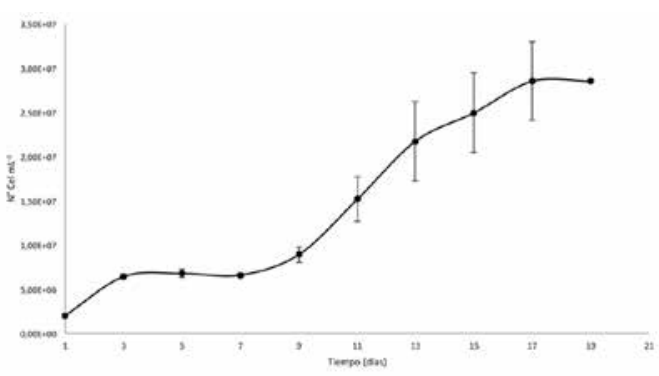

Fuente: autores.

Fig. 12. CURVA DE CRECIMIENTO DE NIVEL DE PH 7.5

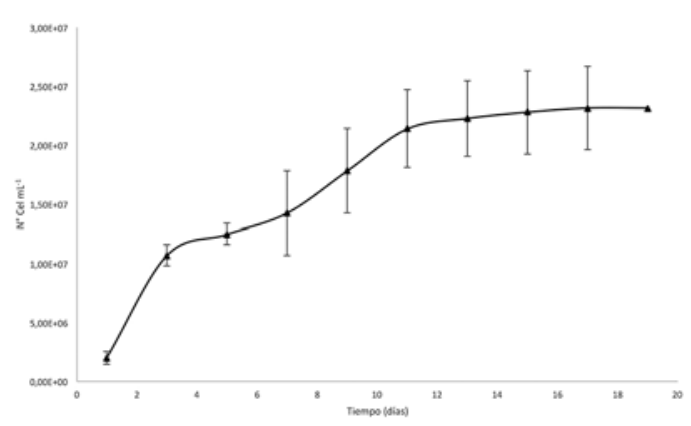

Fuente: autores. 
La productividad volumétrica de cada una de las muestras se calculó utilizando la ecuación (5), los resultados se reportaron en la Tabla V.

TABLA V

PRODUCTIVIDADES VOLUMÉTRICAS DE CONCENTRACIONES SALINAS Y NIVELES DE pH

\begin{tabular}{|c|c|}
\hline Concentración salina & $\begin{array}{c}\text { Productividad volumétrica } \\
{[\mathrm{g} / \mathrm{L} \text { *ía }]}\end{array}$ \\
\hline $0.2 \mathrm{M}$ & 0.53 \\
\hline $0.3 \mathrm{M}$ & 0.45 \\
\hline Nivel de $\mathrm{pH}$ & 1.10 \\
\hline 7 & 0.10 \\
\hline 7.5 & \\
\hline
\end{tabular}

Fuente: autores.

La Tabla $V$ señala que la concentración salina de $0.2 \mathrm{M}$ presentó mayor productividad, al igual que el nivel de $\mathrm{pH}$ 7. Quedando descartadas las demás variables, debido a que la productividad que se obtuvo fue menor.

Por otro lado, se comparó el valor de la productividad más alta que en este caso es el nivel de pH 7 (Tabla III) con respecto a otra productividad de la misma especie de cepa que en este caso es Chlorella y cultivada a un volumen de $400 \mathrm{~L}$ en un fotobiorreactor de placa plana. Esta investigación fue realizada por el Colegio Universitario de Dublín en donde reportaron un valor de productividad de 3.2 g / L*día [19], lo que se determinó como un buen indicio debido a que se tuvo que cultivar a grandes volúmenes para obtener un valor de productividad volumétrica considerable. Mientras que en este trabajo de grado la productividad que se obtuvo fue aproximadamente la tercera parte de la productividad volumétrica registrada en el artículo, pero a un volumen de trabajo $1.4 \mathrm{~L}$.

El análisis estadístico que se desarrolló en esta sección fue una tabla ANOVA de dos factores, el cual por medio de los valores calculados en las curvas de crecimiento se comparó qué variable presenta mayor incidencia si es la salinidad o el $\mathrm{pH}$. Los resultados mostraron que el $\mathrm{pH}$ es la variable que incide en un cultivo de microalgas, debido a las diferencias entre cada uno de los tres criterios con respecto a las concentraciones salinas. Con esta herramienta adicionando las curvas de crecimiento y las productividades volumétricas se seleccionó el nivel de $\mathrm{pH} 7$ como muestra para los análisis de perfil de ácidos grasos mediante cromatografía de gases.
Dicha muestra fue llevada a los laboratorios de toxicología de la Universidad Nacional de Bogotá en el que se obtuvieron los siguientes perfiles Fig. 13 y Fig 14.

Fig. 13. PERFIL DE ÁCIDOS GRASOS A pH 7

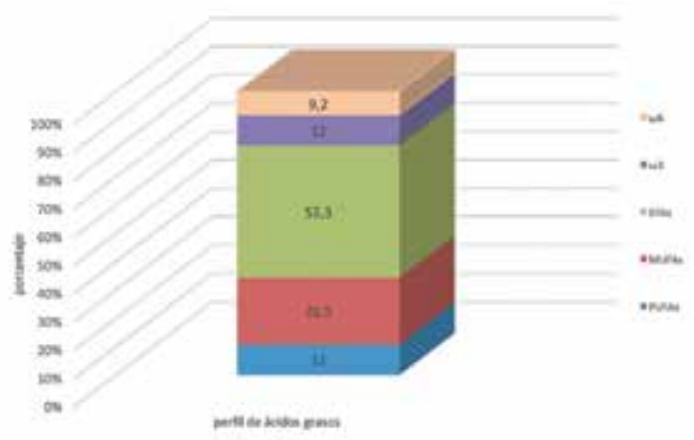

Fuente: autores.

La biomasa de $\mathrm{pH} 7$ obtenida muestra que tiene un porcentaje de $52.3 \%$ de ácidos grasos libres o SFAs, esto quiere decir que esta condición favorece en la obtención de materia prima para combustibles alternativos. Los porcentajes de los omegas resultan ser bajos, por lo que no se recomendaría utilizar esta biomasa con fines alimenticios.

Fig. 14. COMPOSICIÓN DE ACIDOS GRASOS pH 7

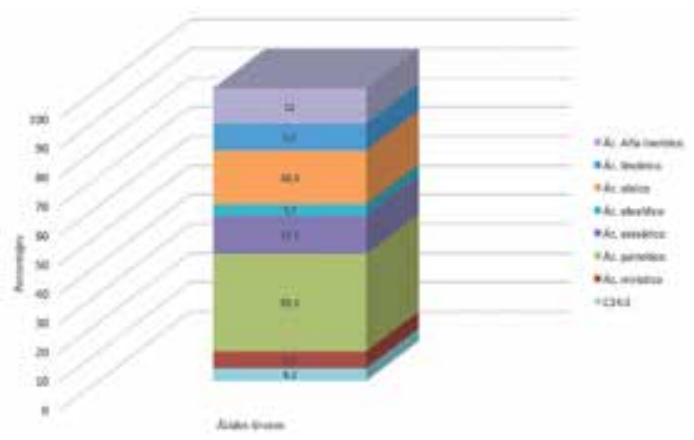

Fuente: autores.

El perfil mostrado en la Fig. 14 confirma que la aplicación de esta muestra es para combustibles alternativos debido al alto porcentaje de ácido palmítico, esteárico y oleico los cuales son materias primas fundamentales en la obtención de biocombustibles.

Por último, se realizaron algunas comparaciones de perfiles de ácidos grasos obtenidos en investigaciones anteriores con los de esta investigación, los resultados se muestran en la Fig. 15 
Fig. 15. COMPARACIÓN DE LíPIDOS ENTRE DOS CEPAS Chlorella

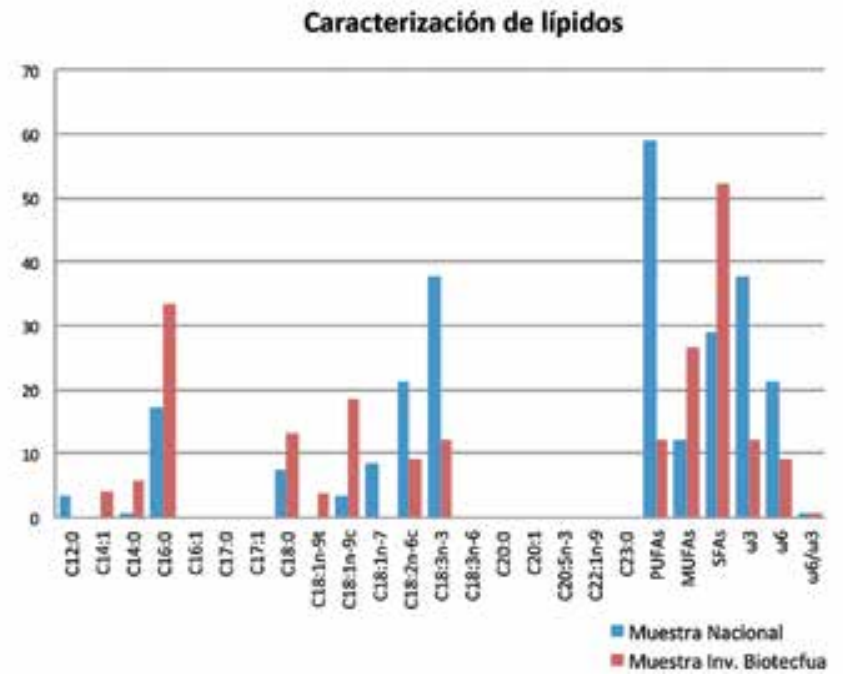

Fuente: autores.

Se compararon los perfiles de ácidos grasos de la misma especie de microalga, el perfil de barras azules fue obtenido en una investigación de la Universidad Nacional de Bogotá [20], y el naranja son los resultados de esta investigación. Como se muestra en la gráfica, en la cepa de la Universidad Nacional el perfil de ácidos grasos es idóneo para la industria de alimentos, gracias a sus composiciones altas de omegas y ácidos esenciales, mientras que la de Biotecfua presenta altas composiciones de ácido palmítico, esteárico y oleico fuentes de materia prima para combustibles alternos.

En la Fig. 16. se muestra una comparación de diferentes especies de microalgas, tales como
Chlorella Vulgaris, Dunaliella, y Scenedesmus obliquus [21], se comparó la cantidad lipídica entre la cepa Dunaliella, y la cantidad lipídica de la cepa que se estudió en esta investigación, y se pudo apreciar que la mayor composición de ácidos grasos fue en la de omega 3 con un 30\% mientras que las demás composiciones de ácidos grasos para biocombustibles son muy reducidas, por lo que no es recomendable utilizar esta cepa como fuente de combustible alterna. Por último, se comparó con la cepa Scenedesmus, en donde su mayor composición lipídica se centró en el omega 6, pero aun así la composición de ácidos grasos para biocombustibles es reducida.

Fig. 16. COMPARACIÓN DE LÍPIDOS DE DIFERENTES ESPECIES DE MICROALGA

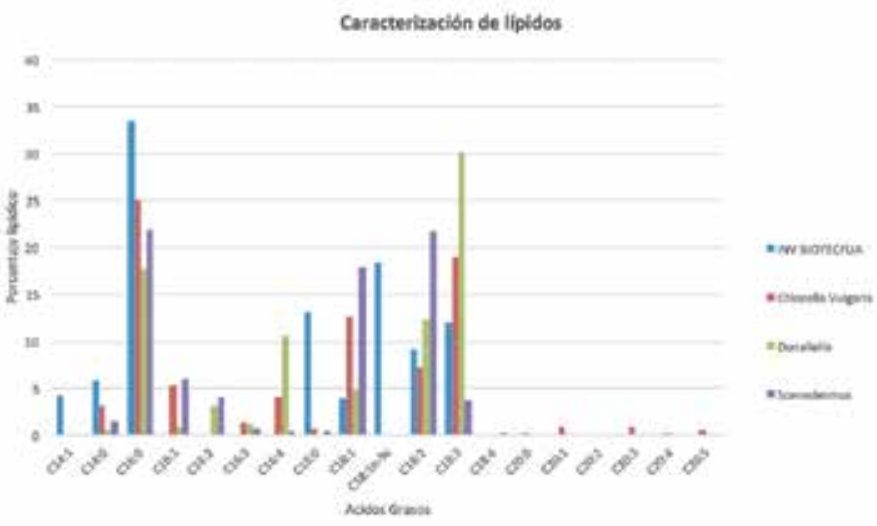


Si queremos encontrar una aplicación rentable en la industria para las dos cepas comparadas sería la de los alimentos, debido a que su porcentaje de ácidos esenciales es alto con respecto a la cepa estudiada. El pH 7.0 se seleccionó como factor base de productividad a nivel de biomasa, es decir, la variable que se tuvo en cuenta en el presente estudio para los cultivos definitivos en fotobiorreactor fue la producción de biomasa. Sin embargo, hay que tener en cuenta que existe un balance entre la cantidad de biomasa producida por los cultivos microalgales y el nivel de lípidos almacenados por estos. En otras palabras, obtener una cantidad óptima de biomasa y lípidos en cultivos es un factor que en la actualidad presenta oportunidades de investigación y consideramos la concentración de biomasa como un indicador adecuado para definirlas como variables de productividad.

El alcance del trabajo nos permitió aproximarnos al efecto de la salinidad y el $\mathrm{pH}$ como factores que pueden modificar la cantidad y el tipo de lípidos obtenidos a partir de C. vulgaris; sin embargo, no nos fue posible generar a partir de estos factores condiciones de estrés que son los que aumentan la producción de lípidos microalgales, en parte, debido a los cambios físicos que sobre el cultivo mismo pueden generan niveles de $\mathrm{pH}$ extremos ácidos o básicos.

\section{CONCLUSIONES}

Tras la experimentación a escala laboratorio con un fotobiorreactor de panel plano para la evaluación de las incidencias de salinidad y $\mathrm{pH}$ sobre la biomasa, productividad y acumulación de lípidos en cultivos de Chlorella vulgaris se pueden obtener las siguientes conclusiones:

Durante la pre-experimentación la variable de salinidad influye en el crecimiento de manera desfavorable debido a que esta especie de microalga es poco halotolerante, es decir, que a concentraciones salinas bajas el inóculo incrementa su concentración celular alcanzando un máximo valor de $1.1 \times 10^{7}$ células por mililitro en la concentración de $0.2 \mathrm{M}$, mientras que si se compara con el nivel de $\mathrm{pH}$ este tipo de microalga se adecúa a niveles parcialmente ácidos a neutros, en este caso el nivel de $\mathrm{pH}$ de 7 reporta un máximo valor de $3.9 \times 10^{7}$ células por mililitro, lo cual es una diferencia significativa.

El fotobiorreactor de placa plana es uno de los equipos adecuados para el cultivo de microalgas, debido a la relación superficie volumen que reportó dicho equipo, la cual fue de $50.1042 \mathrm{~m}^{-1}$. Cumpliendo con los intervalos propuestos en investigaciones anteriores, permitió que el inoculo realice sin problemas el proceso de la fotosíntesis sin llegar a la fotoinhibición.

La máxima concentración alcanzada en cada uno de los ensayos de salinidad (0.2 y $0.3 \mathrm{M})$ corresponde a $1.25 \times 10^{7}$ células por mililitro y $1.03 \times 10^{7}$ células por mililitro. Mientras que los niveles de $\mathrm{pH}$ ( 7 y 7.5 ) reportaron unos valores de $2.85 \times 10^{7}$ células por mililitro y $2.32 \times 10^{7}$ células por mililitro. Lo que evidencia el buen funcionamiento del fotobiorreactor y a la vez permite corrobar que el crecimiento de la biomasa disminuye al aumentar la concentración salina.

La productividad volumétrica del sistema de producción de biomasa se ve afectada tanto para las concentraciones salinas $(0.2$ y $0.3 \mathrm{M})$ como para los niveles de $\mathrm{pH}$, debido a que esto reduce la división celular dando como resultado productividades de 0,526 y 0,421 g/L*día. Caso contrario presentó los niveles de pH (7 y 7.5) que estimularon la división celular dando como resultados de productividad de 1.090 y $0.978 \mathrm{~g} / \mathrm{L}$ *día.

Los resultados obtenidos sugieren que las condiciones experimentales estudiadas, niveles de $\mathrm{pH}$ y salinidad en Chlorella vulgaris pudieron haber causado cambios en el contenido lipídico de la microalga, ya que las mayores composiciones fueron ácidos grasos libres (SFAS) 52,3\% y ácidos grasos monosaturados (MUFAS) 26,5\%, resultados que tienden a ser diferentes de los lípidos obtenidos con $C$. vulgaris lo que también es verificable por la cantidad de ácidos grasos poliinsaturados (PUFAS) obtenidos que se disminuyó considerablemente en un $12 \%$.

Dadas las características químicas de los lípidos obtenidos en el presente estudio, surge la perspectiva real de utilizar los lípidos microalgales para biocombustibles como el biodiesel; sin embargo, las posibilidades de generar producciones industriales se podrán dar a partir de la integración con biorrefinerías [22], las cuales permiten obtener las materias primas necesarias para los 
cultivos de forma sostenida y a costos relativamente bajos. Sin embargo, considerando la mayoría de estudios de obtención de lípidos microalgales y la demanda de alimentos como los aceites omega tres y seis, es posible que las microalgas como Chlorella vulgaris sean más valiosas para este tipo de producciones que para la industria de los biocombustibles. Para definir este tipo de aspectos desde el punto de vista técnico se requieren otros estudios que permitan obtener conclusiones que planteen las producciones industriales [23], [24].

\section{AGRADECIMIENTOS}

Los autores agradecen a las personas e instituciones que apoyaron la presente investigación, especialmente a los padres de Alejandro y a la Dirección de Investigación de la Universidad de América.

\section{REFERENCIAS}

[1] C. C. Flores, J. Mario, P. Castro, L. Bernardo, F. Cotera, R. O. Cañizares, C. Contreras-flores, J. M. Peña-castro, L. B. Flores-cotera, and R. O. Cañizares-villanueva, "Redalyc.Avances en el diseño conceptual de fotobiorreactores para el cultivo de microalgas," Red Rev. Cient. Ámerica Lat., vol. 28, no. 8, p. 8, 2003.

[2] C. Ángel and W. Pimienta, "Evaluación de la incidencia del nitrógeno en el medio de cultivo bold basal para la producción de biomasa de la microalga chlorella vulgaris en un fotobiorreactor, para la obtención de ácidos grasos," Fundación universidad américa, 2012.

[3] A M. Santos, M. Janssen, P. P. Lamers, W. a C. Evers, and R. H. Wijffels, "Growth of oil accumulating microalga Neochloris oleoabundans under alkaline-saline conditions.," Bioresour. Technol., vol. 104, pp. 593-9, Jan. 2012.

[4] N. York, "Effects of pH on growth, photosynthesis, respiration, and copper tolerance of three Scenedesmus strains," vol. 37, pp. 153-160, 1997.

[5] S. Hiremath and P. Mathad, "Impact of Salinity on the Physiological and Biochemical Traits of Chlorella," vol. 1, no. 2, pp. 51-59, 2010.

[6] A. Ruiz, "Puesta en marcha de un cultivo de microalgas para la eliminación de nutrientes de un agua residual urbana previamente tratada anaeróbicamente.," Tesis de Maestría Univ. Politécnica Val., p. 102, 2011.

[7] K. C. Solano and J. O. A. Elkin, "Determinación de la incidencia de la relación carbono: nitrógeno en la producción de biomasa y ácidos grasos en la microalga
Chlorella vulgaris a escala de laboratorio.," Fundación Universidad América, 2013.

[8] J. R. Benavente-Valdés, J. C. Montañez, C. N. Aguilar, A. Méndez-Zavala, and B. Valdivia, "Tecnología de cultivo de microalgas en fotobiorreactores," Rev. Científica la Univ. Autónoma Coahuila, vol. 4, no. 7, p. 12, 2012.

[9] D. Rubio, C. Alcalá, and G. Ingrid, "Evaluación de la incidencia de la temperatura en la producción de lípidos obtenidos a partir de biomasa microalgal a escala laboratorio," Rev. Investig. Univ. América, vol. 6, no. 2, p. 13, 2013.

[10] O. Bastidas, "Conteo Celular con Hematocitómetro," Tech. Note - Neubauer Chamb. Cell Countign, vol. 1, no. 1, pp. 1-6, Apr. 2011.

[11] D. C. Montgomery, Diseño y analisis de experimentos, 2nd ed. México, 2005.

[12] C. Wang, H. Li, Q. Wang, and P. Wei, "Effect of pH on growth and lipid content of Chlorella vulgaris cultured in biogas slurry.," Sheng Wu Gong Cheng Xue Bao, vol. 26, no. 8, pp. 1074-9, Aug. 2010.

[13] J. Sierra and S. Ruiz, "Evaluacion de un fotobiorreactor a escala banco para el cultivo de microalgas y la generación de biomasa.," Fundación Universidad de América, 2012.

[14] D. Rubio, F. María, C. Ángel, and W. Pimienta, "Incidencia del nitrógeno en la producción de biomasa y ácidos grasos de la microalga Chlorella vulgaris en un fotobiorreactor de panel plano a escala laboratorio," Rev. Investig. Univ. América, vol. 6, no. 1, pp. 7-18, 2013.

[15] L. E. Bermero Castillo and J. A. Vargas Machuca, "Estudio del cosechado de cultivos de microalgas en agua residual mediante técnicas de centrifugado," universidad técnica particular de loja, 2011.

[16] J. U. Grobbelaar, "Mass Production of Microalgae at Optimal Photosynthetic Rates."

[17] M. R. Tredici, N. Bassi, M. Prussi, N. Biondi, L. Rodolfi, G. Chini Zittelli, and G. Sampietro, "Energy balance of algal biomass production in a 1-ha 'Green Wall Panel' plant: How to produce algal biomass in a closed reactor achieving a high Net Energy Ratio," Appl. Energy, vol. 154, pp. 1103-1111, 2015.

[18] M. Takagi and T. Yoshida, "Effect of Salt Concentration on Intracellular Accumulation of Lipids and Triacylglyceride in Marine Microalgae Dunaliella Cells," vol. 101, no. 3, pp. 223-226, 2006.

[19] L. Brennan and P. Owende, "Biofuels from microalgae-A review of technologies for production, processing, and extractions of biofuels and co-products," Renew. Sustain. Energy Rev., vol. 14, no. 2, pp. 557-577, Feb. 2010. 
[20] L. M. Serrano Bermúdez, "Estudio de cuatro cepas nativas de microalgas para evaluar su potencial uso en la producción de biodiesel," Universidad Nacional de Colombia, 2012

[21] L. Gouveia and A. C. Oliveira, "Microalgae as a raw material for biofuels production.," J. Ind. Microbiol. Biotechnol., vol. 36, no. 2, pp. 269-74, Feb. 2009.

[22] M. L. Gerardo, S. Van Den Hende, H. Vervaeren, T. Coward, and S. C. Skill, "Harvesting of microalgae within a biorefinery approach: A review of the developments and case studies from pilot-plants," Algal Res., vol. 11, pp. 248-262, 2015.
[23] S. K. Wang, Y. R. Hu, F. Wang, A. R. Stiles, and C. Z. Liu, "Scale-up cultivation of Chlorella ellipsoidea from indoor to outdoor in bubble column bioreactors," Bioresour. Technol., vol. 156, pp. 117-122, 2014.

[24] X. Zhou, L. Xia, H. Ge, D. Zhang, and C. Hu, "Feasibility of biodiesel production by microalgae Chlorella sp. (FACHB-1748) under outdoor conditions," Bioresour. Technol., vol. 138, pp. 131-135, 2013. 